Widefield ScIENCE AND TeChNOLOGY For the SKA

SKADS CONFERENCE 2009

S.A. Torchinsky, A. van Ardenne, T. van den Brink-Havinga, A.J.J. van Es, A.J. Faulkner (eds.)

4-6 November 2009, Château de Limelette, Belgium

\title{
EMBRACE Local Oscillator distributor *
}

\author{
G. Bianchi ${ }^{1}$, J. Morawietz ${ }^{2}$, S. Mariotti ${ }^{1}$, F. Perini ${ }^{1}$, M. Schiaffino ${ }^{1}$, and G. W. Kant ${ }^{2}$
}

1 I.N.A.F. - I.R.A, Via Fiorentina, 3508/B 40059 Medicina, Italy email: g.bianchi@ira.inaf.it, s.mariotti@ira.inaf.it, f.perini@ira.inaf.it, mschiaffino@med.ira.inaf.it

2 ASTRON Oude Hoogeveensedijk 47991 PD Dwingeloo, The Netherlands email: morawietz@astron.nl,kant@astron.nl

\begin{abstract}
EMBRACE (Electronic Multi-Beam Radio Astronomy ConcEpt) has been planned as about 300 square meters aperture array with multiple large independent Field of View (FoV) capabilities. The final design incorporates 220 double conversion receivers. This implies that the system needs two LO (Local Oscillator) distribution networks. In this contribution both the design and the final implementation of the two LO distributor systems are reported. Since EMBRACE operates as a phased array, it is extremely important to guarantee the same phase relation among the receivers. For this reason, a study and a measurement of the LO distributor phase noise were performed and reported in this paper.
\end{abstract}

\section{Introduction}

EMBRACE operates in the frequency band 400-1600 MHz and it has two independent and steerable beams. In order to obtain that feature, the analogue receiver chains are split by 2 somewhere at the tile level, just before the first beam former stage. Due to its large operational bandwidth, the receiver chains adopt a double conversion scheme (Monari et al. 2008), see Fig. 1; this implies that the system needs two Local Oscillators (LO). The first conversion shifts the RF band to the first intermediate frequency $\left(1^{\mathrm{st}} \mathrm{IF}\right)$, centred at $3000 \mathrm{MHz}$ and $100 \mathrm{MHz}$ wide. This operation requires a variable LO whose frequency sweeps from $1400 \mathrm{MHz}$ to $2600 \mathrm{MHz}$. The second conversion shifts $1^{\text {st }} \mathrm{IF}$ to the second intermediate frequency $\left(2^{\text {nd }} \mathrm{IF}\right)$ at $150 \mathrm{MHz}$ (i.e. $2^{\text {nd }} \mathrm{IF}$ band is $100-200 \mathrm{MHz}$ ). This operation requires a fixed LO at $2850 \mathrm{MHz}$. Both LOs must drive mixers located in the receiver boards (see Fig. 2), named Control and Down Conversion unit (CDC), where the requested input power level is in the range between +2 to $+5 \mathrm{dBm}$.

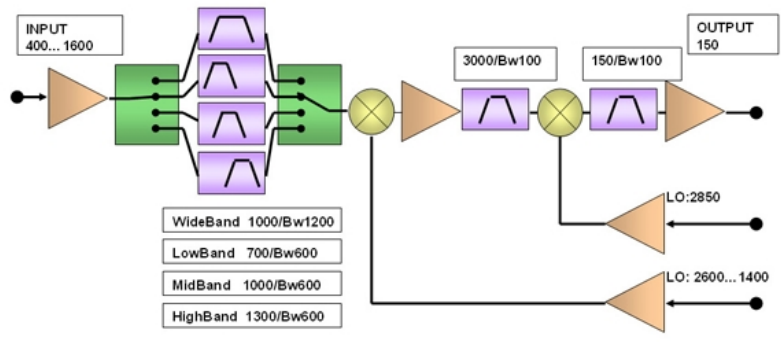

Fig. 1: EMBRACE receiver general architecture.

\footnotetext{
^ This work was supported by the European Commission Framework Program 6, Project SKADS, Square Kilometre Array Design Studies (SKADS), contract no 011938.
}

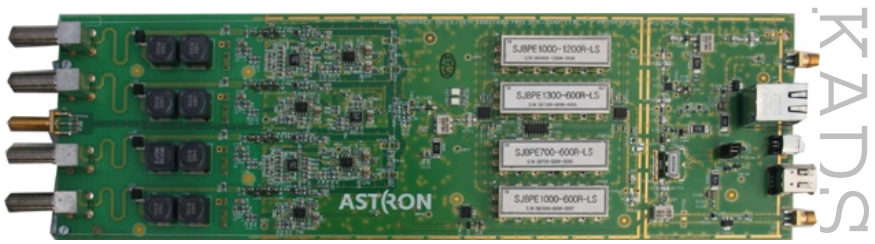

Fig. 2: CDC board.

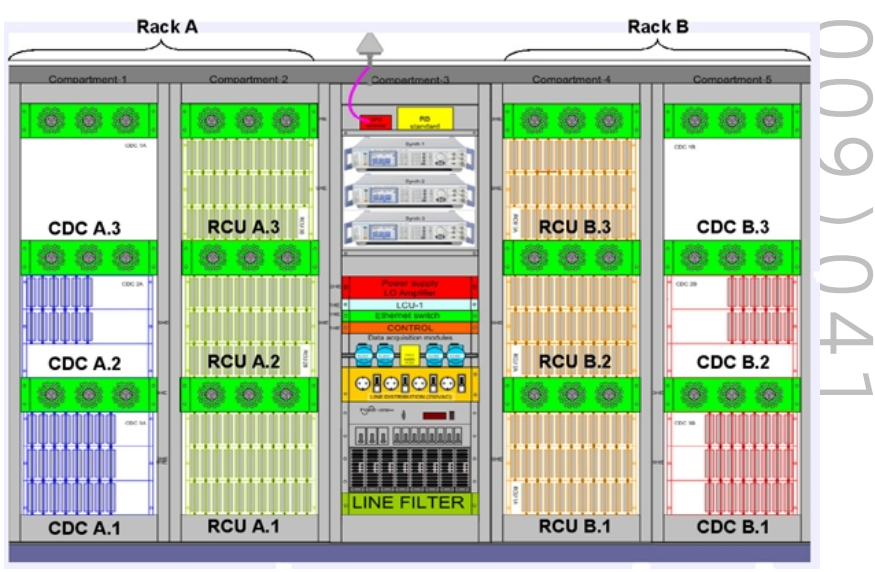

Fig. 3: Racks within the WATS container.

\section{System description}

Since EMBRACE works as a phased array, it's extremely important to guarantee the same phase relation among the receivers. For that reason, to distribute the LOs from the synthesizers to the receiver boards, a splitter chain architecture has been planned for each LO chains (Bianchi et al. 2008). In Fig. 3 is reported a sketch of the planned LO distribution system within the racks in the Westerbork Array Test Station (WATS) container. There are five racks. The central one is equipped with power supply, GPS receiver and also contains the three synthesizers and the LO-amplifiers. "Rack A", on the 


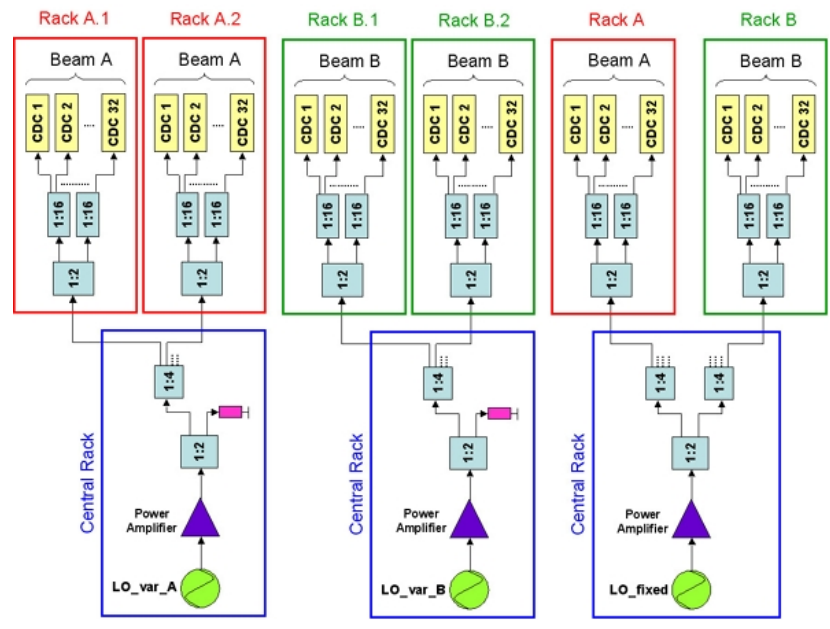

Fig. 4: LO splitter chain distributor.

left, contains the ReCeiver Unit (RCU) boards and the CDC units for beam A and "Rack B", on the right, the same for beam B. Both racks consist of three sub-racks which one can contain up to $32 \mathrm{CDCs}$ and the associated RCUs. Beams A and B have distinct first variable LOs (LO_var_A and LO_var_B) and a common fixed second LO (LO_fixed). To obtain the hardware compatibility among the three LO chains, the system has been designed with the same architecture for all the LOs (Fig. 4). Some splitter outputs are free for possible future expansions of the system (at this level they are closed with $50 \Omega$ loads). In order to design the system, a market survey has been done for each component of the splitter chain. Regarding the synthesizer, the Rohde\&Schwarz SMA100A, with option B22 (lower phase noise specification), has been chosen for its good performance and reasonable costs. Its maximum output power level is $+18 \mathrm{dBm}$. Considering the number of the levels of the splitter chian and the attenuation of filters and cables, a minimum power of $+34 \mathrm{dBm}$ at the beginning of the chain is required. For that reason a high power amplifier is needed since it should operate well below its $1 \mathrm{~dB}$ compression level $\left(\mathrm{P} 1 \mathrm{~dB}_{\text {out }}\right)$. This should be true also for all the other devices which compose the splitter chain, but the active devices, as the amplifiers, are the most critical ones for the generation of unwanted harmonics and the AM to PM conversion, when working close to the compression point. The selected amplifier has been the AM003040SF-2H from Amcom. It guarantees an output power level of $+40 \mathrm{dBm}\left(\mathrm{P} 1 \mathrm{~dB}_{\text {out }}\right)$ with $21 \mathrm{~dB}$ of gain in the $1400-2850 \mathrm{MHz}$ band. This means the amplifier works $6 \mathrm{~dB}$

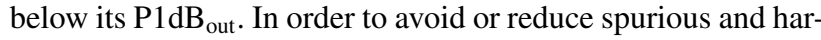
monic components, it is extremely important to put a filter at the output of the high power amplifier. Due to the difference in the operative frequency band of the two LO chains, different filtering design considerations have been done. In the LO_fixed chain, the VLF-3000 filter from Mini-Circuits has been chosen. Concerning the variable LO chain, the choice has been for the VLF-2500 filter from Mini-Circuits. The most critical devices are the 2-way splitters placed right after the high power amplifier. There the power level is about $+33 \mathrm{dBm}$. The

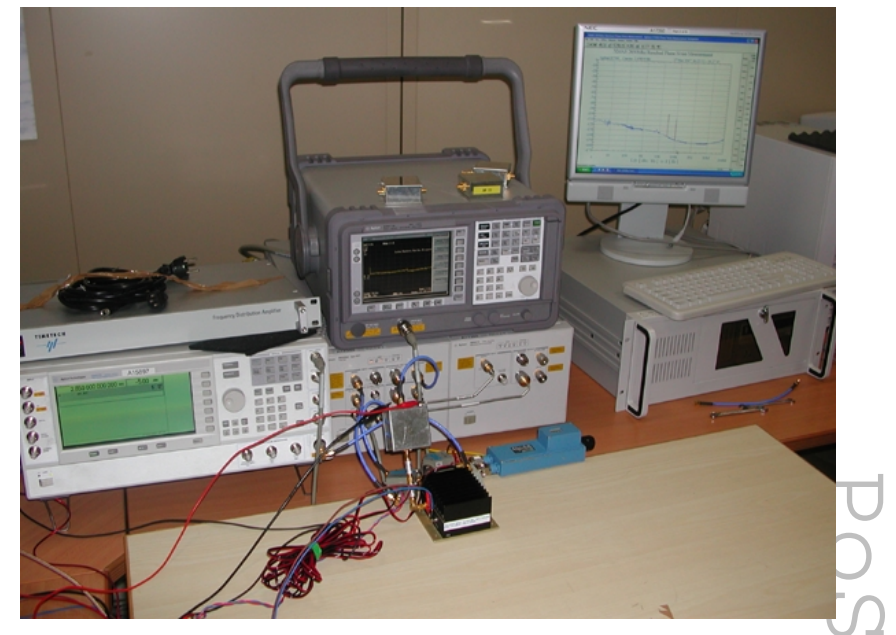

Fig. 5: Phase noise test bench.

ZN2PD2-63+ from Mini-Circuits seems to be the best solution. It works about $7 \mathrm{~dB}$ below its maximum input power level, Regarding the other splitters that compose the chain, the input power is not so critical because they are placed where the power level is lower. For this reason, the model ZN2PD2-63+ (2-way) and the model ZN4PD1-50+ (4-way) from Mini-Circuits have been selected. At the end of the chain, a 16-way power splitter is needed. An excellent device is the 10A3BBD-16S from Renaissance Electronics Corporation; it presents a very low insertion loss. More details on the done choices are reported in the technical report (Bianchi et al. 2008).

\section{Phase noise measurements}

It is very important that the power amplifiers in the LO splitter chain architecture do not increase the phase noise (Bianchi et al. 2007). Some measurements of the phase noise due to the amplifiers have been performed, in particular:

1. Additive (residual) and absolute (total) phase noise.

2. AM to PM conversion to measure the phase modulation.

The most important one is the splitter chain additive phase noise, because it allows to understand the real contribution of the phase noise introduced by the amplifiers and power splitters. The phase noise measurement it is not easy: it needs extremely precision instruments and care of the test bench. For this reason, an Agilent E5500 Phase Noise Measurement Subsystem for the absolute and residual phase noise measures has been selected (Fig. 5). After the set-up phase, where the instrumental noise has been measured, the additive phase noise of the mixer driver (an HMC480ST89 amplifier from Hittite) was measured at $2850 \mathrm{MHz}$ (maximum input frequency of the system). The phase noise contribution of the amplifier is very low: in comparison to the instrumental noise, we can observe only a negligible noise increment; less than $10 \mathrm{~dB}$ for each frequency point (Fig. 6). Then we measured the additive phase noise due to the whole splitter chain (composed of splitters and mixer driver). In order to simulate the effect of the splitter 


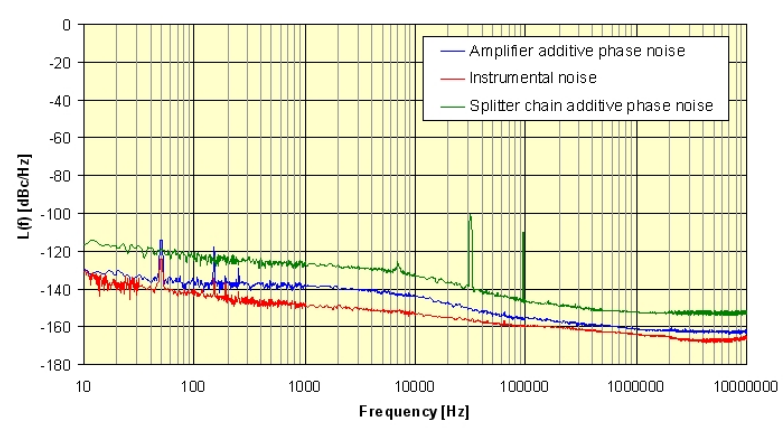

Fig. 6: Additive phase noise comparison measure.

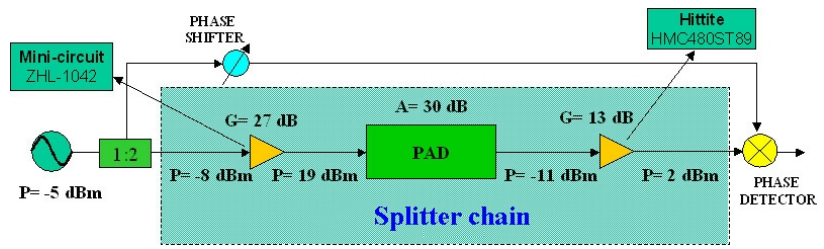

Fig. 7: Additive phase noise measurement: whole splitter chain block diagram.

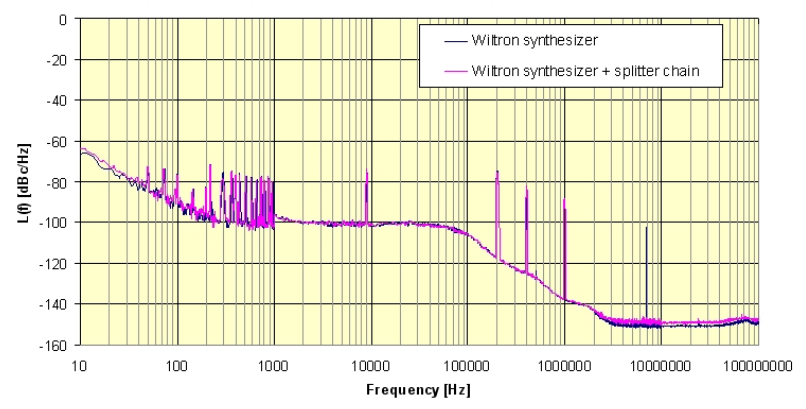

Fig. 8: Absolute phase noise comparison measure.

chain power dividers, $30 \mathrm{~dB}$ attenuators were used as shown in Fig. 7. The result of this measure is reported in Fig. 6 (green line), where an extremely low integrated phase noise value, less than $0.03 \mathrm{deg}$, was found. The pad was also set along the LO distribution chain at different positions (right next the synthesizer and before the mixer), but no meaningful differences were found in the graph shown in Fig. 6. Putting a synthesizer at the beginning of the splitter chain, the absolute phase noise of the whole chain has been measured. In this case, a Wiltron 68159B at $2850 \mathrm{MHz}$ was used. In Fig. 8 is reported the measure of the phase noise of the Wiltron synthesizer alone and the measure of the Wiltron synthesizer plus the splitter chain. As we expected, there is an undetectable difference between the two cases. Therefore, the main contribution of the phase noise is due to the synthesizer: in order to minimize the phase noise of the LO distribution chain, a good synthesizer is required!

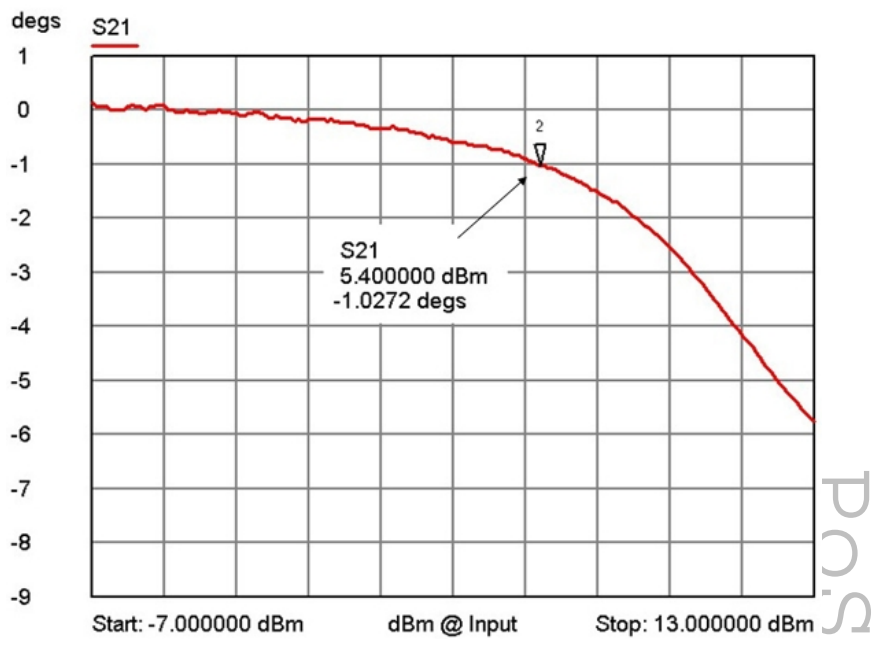

Fig. 9: AM to PM conversion of PA.

\section{AM to PM conversion measure}

The phase modulation (PM), due to the amplitude variation, was generated in different ways in order to make the measurements. A strong enough amplitude variation has been driven to the PA (Power Amplifier) under test (HMC480ST89) in order to excite the non-linear region. Due to the available instrumentation, two methods have been performed:

1. The first has been the classical AM to PM conversion mea surement using, as a stimulus, a large sine wave at the operating frequency.

2. The second has been performed by changing the bias con ditions and the correlated phase modulation has been observed.

Moreover a third test has been conducted: the variation of the phase due to the temperature variations. For the AM to PM conversion measurement, a HP 8753C network analyzer (power sweep mode) has been used. Test and analysis has been conducted in L and S bands. Fig. 9 shows the phase of S21 vs input power. In order to find an indicative quantity to evaluate the $\mathrm{AM}$ to $\mathrm{PM}$ conversion, the $\Delta \Phi / \Delta$ Power has been calculated into a convenient range of power. As may be seen in the Fig. 10, the maximum value of $\Delta \Phi / \Delta$ Power is $0.2 \mathrm{deg} / \mathrm{dB}$. During the measurement of the PA, others sources and causes of PM different from AM to PM classical conversion has been found. More specifically, the supply voltage has been changed around the nominal value and the phase variations have been recorded. Four PAs have been tested and they have showed a phase variation (due to bias condition) of $0.1-0.2 \mathrm{deg} / \mathrm{V}$. Since $1 \mathrm{mV}$ is an usual residual supply ripple, a phase jitter due to bias supply is about $0.0001 \mathrm{deg}$. Past experience has shown a typical behaviour of Gain vs Temperature of a GaAs Amplifier close to $-0.005 \mathrm{~dB} /{ }^{\circ} \mathrm{C}$ every $10 \mathrm{~dB}$ Gain stage. For a PA driven close to non linear region, this variation will produce a variation of the phase that follows the same law described for the AM to $\mathrm{PM}$ conversion. As an example, into a $10 \mathrm{~dB}$ gain $\mathrm{PA}$, providing $+15 \mathrm{dBm}$ at the output, a $10{ }^{\circ} \mathrm{C}$ of temperature variation 


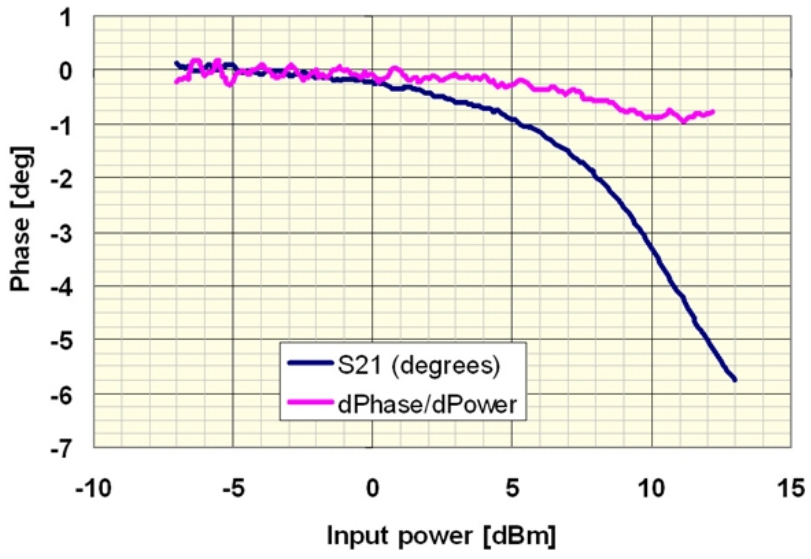

Fig. 10: $\Delta \Phi / \Delta$ Power.

will increase the gain of $0.05 \mathrm{~dB}$ and looking at Fig. 10, the consequently phase variation will be $0.01 \mathrm{deg}$.

\section{PM due to adjacent branches variations}

If one (or more) adjacent LO lines and PA change their own input reflection coefficient due to finite isolation between branches, a phase variation may be observed on the PA under test. This phenomenon can be simulated. The worst case occur for the closest branches (where the isolation is the smallest) and for angles $90^{\circ}$ far. The simulated phase change is: Phase change $=\arctan \left(10^{(\mathrm{RL}+\mathrm{ISO}) / 20}\right)$, where RL is the return loss of adjacent RF path and ISO is the isolation of the two RF paths. As example, for two closest path on splitter chain, $20 \mathrm{~dB}$ isolated, feeding two PA with Input Return Loss of $10 \mathrm{~dB}$, if the adjacent branch change its phase or delay, into the observed branch the phase will change of $1.8 \mathrm{deg}$. While a completely failure of the same adjacent $\mathrm{PA}(\mathrm{RL}=0 \mathrm{~dB})$ will produce a phase error of $5.7 \mathrm{deg}$.

\section{Conclusions}

The design of the EMBRACE Local Oscillator distributor has been realized choosing a splitter chain architecture and choosing best devices in terms of high performance, low phase noise, low cost, low consumption and easy mechanical assembling. The LO distributor has been installed in the racks shown in Fig. 11. The Phase Noise of some Power Amplifiers (used in LO branches) has been measured. The Phase Noise has been measured both in terms of spectral density and integrated over frequency and it has been measured for different chains, including the effect of the strongly attenuating splitter chain. Also, the correlation of phase changes (jitter) has been observed versus temperature, bias voltage and RF power level (also in non linear region). Table 1 summarises the possible causes and the effect observed. For moderate gain Amplifiers like the models tested, the Added Phase Noise, Jitter and Phase Error (due to both exogenous and endogenous causes) are very small. They typically are one or more order of magnitude smaller than the phase jitter generated by other electronic parts, i.e. oscillators

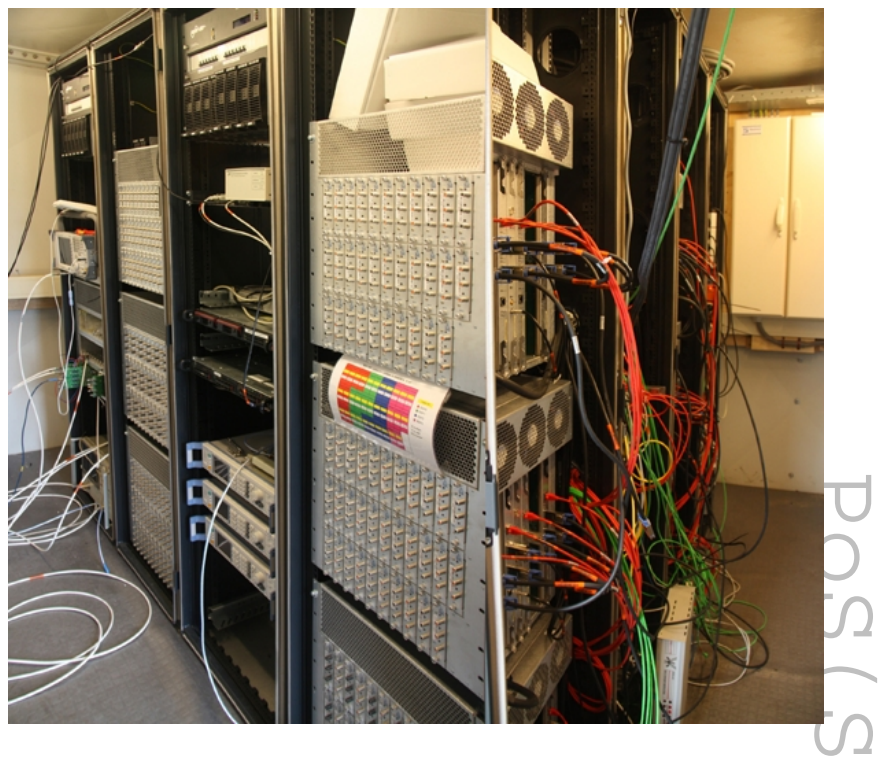

Fig. 11: Photo of the racks in the WATS container.

Table 1: Phase Noise causes and typical values.

\begin{tabular}{|l|c|}
\hline $\begin{array}{l}\text { Integral of Spectral } \\
\text { Phase Noise Density }\end{array}$ & $0.03 \mathrm{deg}$ \\
\hline AM to PM (max value) & $0.2 \mathrm{deg} / \mathrm{dB}$ \\
\hline Jitter due to Bias Supply $(1 \mathrm{mV})$ & $0.0001 \mathrm{deg}$ \\
\hline $\begin{array}{l}\text { Jitter due to Temperature } \\
\text { Variation }\left(10^{\circ} \mathrm{C}\right)\end{array}$ & $0.01 \mathrm{deg}$ \\
\hline $\begin{array}{l}\text { Phase Error due to a strong } \\
\text { change into the adjacent RF path }\end{array}$ & $2 \mathrm{deg}$ \\
\hline
\end{tabular}

or long transmission lines. The added splitter chain phase noise is negligible with respect to the confusion limit.

\section{References}

Monari, J., Perini, F., Mariotti, S. , Montebugnoli, S., Bianchi, G., "EMBRACE Receiver (SKADS Task DS5-T1WP4)," IRA technical report N.414/08, 2008.

Bianchi, G., Morawietz, J., Perini, F., Schiaffino, M., "EMBRACE LO distribution system," IRA technical report N.426/08, 2008.

Bianchi, G., Mariotti, S., Morawietz, J., "Added Phase Noise measurement for EMBRACE LO distribution system," IRA technical report N.407/07, 2007. 